\title{
Stratégies pour Thèbes chez Pindare
}

Strategies for Thebes in Pindar

\section{André Hurst}

\section{OpenEdition}

Journals

Édition électronique

URL : https://journals.openedition.org/gaia/391

DOI : 10.4000/gaia.391

ISSN : 2275-4776

\section{Éditeur}

UGA Éditions/Université Grenoble Alpes

\section{Édition imprimée}

ISBN : 978-2-37747-057-0

ISSN : 1287-3349

\section{Référence électronique}

André Hurst, «Stratégies pour Thèbes chez Pindare », Gaia [En ligne], 21 | 2018, mis en ligne le 01 novembre 2018, consulté le 10 décembre 2021. URL : http://journals.openedition.org/gaia/391 ; DOI : https://doi.org/10.4000/gaia.391

Ce document a été généré automatiquement le 10 décembre 2021.

Gaia. Revue interdisciplinaire sur la Grèce archaïque 


\title{
Stratégies pour Thèbes chez Pindare
}

\author{
Strategies for Thebes in Pindar
}

André Hurst

\section{NOTE DE L'AUTEUR}

Je remercie les deux réviseurs anonymes de cette contribution pour leurs judicieuses remarques. J'en ai tenu compte dans toute la mesure du possible. Je porte évidemment l'entière responsabilité des imperfections.

1 À l'issue des guerres médiques, Pindare se trouve dans une situation embarrassante : sa cité de Thèbes est indéniablement "du mauvais côté ${ }^{1}$. Thèbes a pris le parti des envahisseurs. L'oracle de Delphes également, avec lequel Pindare entretient des rapports privilégiés. Cependant, si le sanctuaire de Delphes a pour lui son statut d'oracle largement reconnu, et qui peut suffire à la préservation de son image, il n'en va certainement pas de même pour une cité plongée dans les luttes politiques. Pour un poète qui, dès le plus ancien poème conservé $\left(P ., 10,-498^{2}\right)$, s'est placé dans une perspective d'ouverture, le risque de se voir confiné, en sa qualité de Thébain, dans un profil de traître à la cause des Grecs est évidemment redoutable. Par ailleurs, renier sa cité ne pouvait se concevoir ${ }^{3}$. Les stratégies de Pindare pour affirmer simultanément son attachement à la cause des Grecs $^{4}$ et sa fierté d'être thébain méritent d'être examinées à la lumière d'une double question : tout à la fois le bon usage du poème de circonstance et la présence d'une «voix personnelle» du poète. Le premier aspect concerne le destinataire ou son commanditaire (dans les cas où ils sont distincts) et le public, le second touche la position personnelle de l'auteur. Poèmes de circonstances, les épinicies de Pindare présentent clairement une façade qui satisfait à la demande du commanditaire ; cependant, le poète est tout aussi présent que le commanditaire, et les soucis du poète peuvent être perçus selon diverses clefs de lecture. C'est ainsi que l'on peut être à l'écoute d'une sorte de plaidoyer pour Thèbes dans l'œuvre de Pindare, un plaidoyer qui procède par touches plus ou moins explicites. Une telle écoute implique évidemment que l'on se situe bien souvent en marge de l'objectif premier du poème, 
que l'on procède, par une sorte de tangente thébaine, au relevé de traits jugés pertinents. Elle repose en outre sur une hypothèse : les mentions de Thèbes ou de faits thébains ne seraient pas «innocents», ils charrieraient une intention apologétique. Cette hypothèse, il est vrai, peut être contestée dans son fondement. Il vaut cependant la peine d'examiner si l'éclairage qu'elle offre n'est pas de nature à faire apparaitre des procédés utilisés par le poète. On pourrait ainsi tenter de déceler, quel que soit leur degré plus ou moins conscient (d'ailleurs invérifiable) des "stratégies pour Thèbes " dans les épinicies de Pindare ${ }^{5}$.

Est-il légitime de supposer que Pindare se soit préoccupé tout au long de sa carrière de la réputation de Thèbes, un peu comme s'il était un ambassadeur de bonne volonté chargé de veiller à l'image de sa cité dans toute la mesure où cette image avait été ternie durant les guerres médiques?

3 Si l'on convient que poser cette question est justifiable, on tiendra compte cependant $\mathrm{du}$ fait que les circonstances évoluent. Il est probable que l'emprise d'Athènes devint après les guerres médiques une préoccupation plus sérieuse dans le monde grec que le souvenir du mauvais choix opéré par Thèbes. Pour autant, cela ne saurait conditionner automatiquement ce qui relève des sentiments, qu'il s'agisse de ceux du poète ou de ceux de tout un chacun. Il est exact aussi que Thèbes conclut des alliances qui montrent que la cité finit par jouir d'une réhabilitation à tout le moins pragmatique. Cependant, les épinicies qui peuvent être datées de décennies postérieures à celle qui suivit la fin des guerres médiques se situent chronologiquement entre - 468 et -444, une période qui n'est pas bien longue : c'est le temps durant lequel on voit grandir principalement la génération des enfants de ceux qui ont combattu lors des guerres médiques ${ }^{6}$.

De son côté, Pindare n'avait-il pas pour préoccupation principale sa propre réputation et les commandes prestigieuses qu'elle pouvait lui valoir? Outre le fait qu'il n'est pas incompatible d'avoir souci de sa carrière et, simultanément, de l'image de sa cité, on rappellera l'anecdote célèbre selon laquelle Pindare fut mis à l'amende par les Thébains pour avoir chanté l'éloge d'Athènes ${ }^{7}$. On se souvient qu'à cette occasion, les Athéniens auraient acquitté le montant de l'amende. L'existence même de cette anecdote est un gage de l'importance que l'on attribuait au rapport des Thébains et de la poésie de Pindare, elle suppose une vigilance de leur part exercée sur sa production et, partant, on est en droit de postuler chez Pindare une sensibilité particulière lorsque l'image de Thèbes est en jeu.

5 On choisit ici de s'en tenir aux épinicies, à l'exclusion des poèmes conservés de manière fragmentaire. Ce choix s'explique par la recherche de stratégies textuelles, donc de manières de composer qui ne peuvent être valablement observées que dans le cadre de textes transmis comme intégralement conservés.

6 Une dernière remarque préliminaire concerne le caractère limité de notre perception des épinicies pindariques: il nous manque deux éléments essentiels, à savoir la musique et la chorégraphie. On sait à quel point la musique peut être porteuse d'allusions ou de rappels, et l'on peut en dire tout autant de la gestique d'une chorégraphie ${ }^{8}$. C'est une limitation dont il convient d'être conscient, même s'il n'est pas vraiment possible d'y porter remède ${ }^{9}$. 


\section{Poèmes célébrant des vainqueurs thébains}

7 On se tournera pour commencer vers les épinicies qui célèbrent des vainqueurs thébains. La glorification de Thèbes, dans ces cas, peut être tenue pour une donnée quasiment obligée, et c'est pourquoi ces épinicies méritent d'être considérées comme relevant d'une catégorie à part.

Une victoire obtenue dans les jeux d'un sanctuaire marque la faveur divine. En ellesmêmes, par conséquent, les victoires des Thébains Mélissos (I., 3+4), Thrasydée (P., 11), Hérodote $(I ., 1)$ et Strepsiade $(I ., 7)$ constituent des moments importants pour qui se soucie de l'image de Thèbes. Dans chacun de ces cas, le rôle du poète consiste à donner un sens à la victoire, un sens qui entraînera presque obligatoirement des éléments touchant à la cité du vainqueur. Que les odes soient exécutées à Thèbes ou ailleurs, l'effet recherché sera présent, même avec des effets divers, car les chants peuvent « prendre le bateau » pour se répandre au loin (N., 5, 2-5).

\section{$-474 / 3 ?-$ Isthmiques 3 et 4}

9 Se trouve-t-on en présence de deux poèmes distincts ou d'un seul ${ }^{10}$ ? Rappelons que si tous deux célèbrent des victoires du Thébain Mélissos, l'un est composé à l'occasion d'une victoire à la course de chars, l'autre pour une victoire au pancrace. Que l'on sépare ou non les deux poèmes, on peut y observer, ensemble ou distribuées, deux procédures complémentaires. Là où la troisième Isthmique procède obliquement, la quatrième attaque la question de front. On constatera que ces deux manières sont présentes dès la huitième Isthmique $(-478$, infra).

Brièveté sublime, ou prélude à de plus amples éloges? Quoi qu'il en soit, la triade qui constituerait à elle seule la troisième Isthmique réunit de manière saisissante une proclamation initiale du nom de Thèbes $(I ., 3,12)$, une allusion cryptée aux vicissitudes du destin à travers la parenté du vainqueur et de la famille célèbre des Labdacides (I., 3 , 16), et une conclusion formulée dans un style énigmatique, venue solliciter l'imagination et dont le sens semble bien être qu'il faut se trouver proche de la condition divine pour ne point subir de revers, ce qui ne saurait être le cas d'une cité comme Thèbes.

11 La proclamation du nom de Thèbes est préparée : le poète la fait précéder par un cortège d'énoncés de valeurs consensuelles. Il présente ce qu'on pourrait appeler un certificat d'inclusion à la pensée morale commune, non sans rappeler que c'est Zeus luimême qui dispense les récompenses à qui les mérite, pour n'en venir qu'ensuite au vainqueur ainsi justifié et, au sommet de l'édifice, à l'affirmation de sa citoyenneté thébaine (I., 3, 1-12). Après la proclamation du nom de Thèbes par le héraut (I., 3, 12), l'occasion est belle d'affirmer que les humains sont sujets à l'erreur: c'est ici qu'intervient le passé thébain. Mélissos, le vainqueur célébré, descend par sa mère des Labdacides. Ce simple nom $(I ., 3,17)$ suffit à évoquer un passé grandiose marqué par deux traits saillants. D'une part la célébrité de la matière de Thèbes permet de faire surgir tout un passé commun du monde grec au travers du seul mot de "Labdacide ", cependant que, d'autre part, ce mot permet opportunément de rappeler que les humains sont parfois jouets d'un destin sur lequel ils n'ont pas de prise :

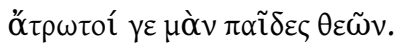


D'invulnérables, il n'est que les enfants des dieux.(I., 3, 18b) ${ }^{11}$ vainqueur, une énumération qui débouche sur l'évocation du destin d'Ajax (I., 4, 22-42). Certes, le poète insiste pour montrer que le destin d'Ajax présente des analogies avec celui des Cléonymides : personnage glorieux, il n'encourt pas moins les reproches des Grecs ${ }^{14}$. Cependant, par la grâce d'un poète, Homère, Ajax retrouve un rang prestigieux (I., 4, 37-42).

17 Le passage de la gloire à l'opprobre pourrait n'être pas évoqué pour le seul cas d'Ajax : la mention de la bataille de Platée permet de faire apparaitre en filigrane le cas de Thèbes dans son ensemble, elle aussi passée de la gloire à l'opprobre, et l'on dépasserait alors le destin d'une seule famille thébaine ${ }^{15}$.

L'action d'Homère rétablissant la renommée entachée d'Ajax pourrait avoir son pendant dans l'intervention à laquelle Pindare lui-même est en train de se livrer en faveur des Cléonymides, une intervention qui, par extension, englobe le cas de Thèbes.

19 Cette préparation débouche sur la mention d'un mérite explicite de Thèbes : elle est la patrie d'Héraclès. La petite taille de Mélissos sert de prétexte à un parallèle avec Héraclès ${ }^{16}$, Héraclès sert à son tour de prétexte à nommer Thèbes une fois encore (I., 4 , 53) dans l'élan d'une phrase qui nous fait aboutir chez les dieux olympiens (I., 4, 55-60). La place d'Héraclès parmi les Olympiens est évidemment un gage de sa valeur pour l'ensemble des Grecs. Au travers de ce choix, ce qui est visé est une sorte de réintégration de Thèbes dans la communauté culturelle grecque. On songe à la manière dont Achille est convoqué en faveur d'Égine dans la huitième Isthmique (infra). 


\section{$-474-$ Pythique 11} d'Oreste, une véritable "Orestie pindarique ${ }^{17}$. Il la fait cependant précéder d'une salve de gloire mythique thébaine, accompagnée d'une évocation de Delphes $(P ., 11,9-10)$, comme il se doit dans une ode pythique (ce premier segment est formé de toute la première triade). On considère généralement que l'insistance du début sur le culte thébain d'Apollon Isménios signale une exécution de l'ode à Thèbes même, voire à l'occasion d'une procession en l'honneur d'Apollon Ismenios (au sanctuaire de qui les filles de Cadmos sont invitées à se rendre dès les premiers vers). Sur ce point, les interprètes ont généralement suivi les scholiastes. On est tenté de penser que l'éloge de Thèbes, directement ou au travers de son citoyen Thrasydée, ne touche pas à la problématique de l'image de Thèbes auprès du reste des Grecs : ce serait en quelque sorte un texte "ésotérique ». Peut-être. Il faut cependant considérer d'abord que les textes voyagent $(N ., 5,1-3)$, puis, en second lieu, que les conseils de modération politique sur lesquels l'ode débouche, adressés à qui veut les entendre, pourraient bien concerner autant les Thébains eux-mêmes que ceux qui critiquaient leur constitution oligarchique au lendemain de l'erreur politique commise durant les guerres médiques ${ }^{18}$.

ix d'Oreste pour la séquence légendaire de l'ode a suscité beaucoup d'ingéniosité chez les critiques. Pindare lui-même lance le défi lorsqu'il pose la question : $\tilde{\eta} \rho$ ', $\tilde{\omega}$

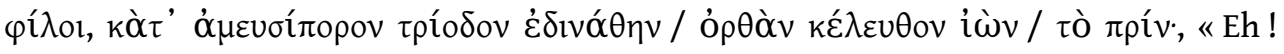
bien, mes amis, me suis-je égaré à ce carrefour, moi qui suivais la bonne route ? [...]» $(P ., 11,38-39)$, et cette provocation n'est pas demeurée sans réponse. Pour les scholiastes, la situation est claire : Pindare s'est égaré et il le reconnaît (schol. ad P., 11, $23 a$ et $58 a)^{19}$. Mais on ne pouvait en rester là ${ }^{20}$. Sans entrer dans les subtilités qui permettraient d'absoudre Pindare d'une accusation d'incohérence, on notera que le lien en apparence ténu qui rattache Oreste à la victoire de Thrasydée existe bel et bien : Strophios, père de Pylade et hôte d'Oreste est phocidien. C'est donc sur ses terres que Thrasydée a obtenu sa victoire ( $P ., 11,12-16)$. S'agit-il d'affirmer un point d'érudition mythologique? C'est peu probable. L'enjeu pourrait être de taille : en introduisant la " matière de Troie » dans la célébration d'un vainqueur thébain, à l'occasion d'une cérémonie typiquement thébaine d'Apollon Isménios, le poète proclame hautement la solidarité de Thèbes avec une tradition légendaire qui touche l'ensemble des Grecs. C'est l'affirmation de la présence de Thèbes dans le concert des cités grecques, malgré les événements guerriers qui se sont déroulés quelques années à peine auparavant ${ }^{21}$.

\section{$-458 ?-$ Isthmique 1}

Écrite pour Hérodote, vainqueur à la course des chars, la première Isthmique s'ouvre sur le nom de Thébé, éponyme de Thèbes, et sur une déclaration d'appartenance très affirmée du poète :

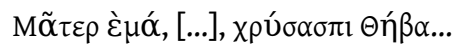

Ma mère, Thébé au bouclier d'or...

Pindare saisit visiblement l'occasion de cette victoire du Thébain Hérodote pour faire entendre bien haut le nom divin qui équivaut à celui de sa cité. Il ne s'agit pas 
simplement de louer un Thébain, mais plus encore d'affirmer le patriotisme thébain du poète: pour servir Thèbes, Pindare affirme qu'il fait passer au second plan une commande des habitants de Céos. Ils attendront, car pour lui, c'est «Thèbes d'abord ". Le reste du poème est parsemé de gloire thébaine, et l'on entend résonner des mots comme «troupe de Cadmos » (I., 1, 11), «enfant d'Alcmène » (I., 1, 12-13), « cours de Dircé » (I., 1, 29 associé à l'Eurotas), « Amphitryon » (I., 1, 55). Quant au nom de Thèbes, il réapparaît non seulement accouplé à celui de Lacédémone dans le corps du poème $(I ., 1,17)$, mais comme par un rappel des premiers mots du texte, on l'entend à nouveau résonner à la fin, cette fois-ci dans la formule traditionnelle "Thèbes aux sept portes "

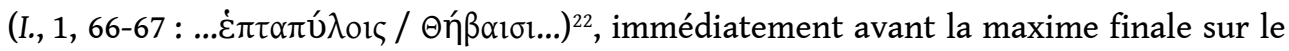
riche avare qui ne connaîtra pas la gloire (I., 1, 67-68).

La partie mythique du poème associe le vainqueur à des hymnes, probablement traditionnels, dédiés à Iolaos et à Castor. Le vainqueur célébré a remporté la victoire à la course des chars et le choix de ces figures mythiques est justifié par le fait qu'il s'agit des deux meilleurs conducteurs de chars " mis au monde à Lacédémone et à Thèbes " $(I ., 1,17)$. S'ensuit un segment dans lequel Sparte et Thèbes sont mises en parallèle au travers de la geste de leurs héros $(I ., 1,18-32)$. Cette partie du texte donne probablement une clef: l'insistance sur Thèbes est certes liée à la personne du vainqueur, mais le rapprochement fortement souligné avec Sparte pourrait s'expliquer par des raisons politiques, en l'occurrence une alliance des deux cités. Dans ce cadre, on observe que Thèbes n'est plus l'objet de stratégies tendant à l'excuser, mais on exhibe au contraire sa gloire comme pour la faire valoir au moment d'un rapprochement souhaitable. Plus encore, le père du vainqueur, Asôpodoros, avait combattu du côté des Perses $^{23}$ lors de la bataille de Platées. Pindare n'hésite pas à évoquer son destin, son exil et sa réhabilitation à Thèbes (I., 1, 34-40). Selon toute apparence, à cette date, plus rien ne s'oppose à ce qu'il chante hautement sa cité natale à la face du monde.

\section{$-454-$ Isthmique 7}

Comme dans la première Isthmique, on trouve le nom de Thébé au premier vers de la septième Isthmique, et ce nom provoque une série d'allusions à la matière de Thèbes ${ }^{24}$. La question initiale (quel est le haut fait du passé auquel Thébé prend le plus de plaisir ?) donne le départ d'un défilé supposé livrer les éléments du choix. On entend ainsi évoquer la naissance de Dionysos (I., 7, 3-5), celle d'Héraclès (I., 7, 5-7), la sagesse de Tirésias (I., 7, 8), Iolaos, l'illustre neveu d'Héraclès $(I ., 7,9)$, les Spartes nés des dents du dragon $(I ., 7,10)$, la guerre des Sept contre Thèbes (I., 7, 10-11), la mission des Égides à Sparte au moment de la guerre d'Amyclées (I., 7, 12-15). L'affirmation traditionnelle que seuls les chants font échapper les hauts faits à l'oubli conclut la série. La suite de l'ode concerne plus particulièrement le vainqueur, Strepsiade, vainqueur au pancrace, et sa famille. Un oncle homonyme est mort à la guerre: c'est l'occasion d'une intervention à la première personne $(I ., 7,37-39)$. Elle fait suite à une déclaration de portée générale sur l'héroïsme de cet oncle tombé au combat (I., 7, 23-36). Dans les mots qui précèdent immédiatement l'intervention à la première personne, Pindare utilise une forme verbale ( $\varepsilon \tau \lambda \alpha \nu, I ., 7,37)$ qui peut exprimer aussi bien une troisième personne du pluriel (« ils souffrirent») qu'une première personne du singulier (" je souffris »), et les interprètes sont partagés sur ce point ${ }^{25}$. En tout état de cause, on concédera qu'il est possible de percevoir, dans l'ombre de la famille du vainqueur, la figure du poète qui eut à souffrir de la guerre et qui, explicitement, a reçu de Poséidon la sérénité après la 
tempête (I., 7, 37-39). Quelle tempête ? S'agit-il de la bataille d'Oïnophyta ? Peut-être ${ }^{26}$. On ne peut s'empêcher cependant de percevoir ici que le temps douloureux des reproches adressés à Thèbes semble désormais révolu ${ }^{27}$.

\section{Poèmes pour des vainqueurs en provenance d'autres cités grecques}

On le constate, l'ensemble des odes destinées à célébrer des vainqueurs thébains date de la période qui suit la fin des guerres médiques. Au moment d'aborder le reste des épinicies, une question de méthode se pose, qui implique la chronologie : à considérer l'ensemble des épinicies, on constate en effet que la matière de Thèbes est présente également dans des odes qui furent écrites avant la fin des guerres médiques. Cependant, à l'évidence, pour qui recherche la réaction possible du poète après le faux pas politique de Thèbes, ce sont les odes écrites après la fin des hostilités qui constituent le champ de l'analyse. Pour autant, les odes qui datent d'avant cette période ne doivent pas être négligées. Elles offrent en effet la possibilité d'une vérification : existe-t-il une différence entre le traitement des thèmes thébains avant et après la fin des guerres médiques? C'est à quoi l'on pourra procéder à la suite de l'examen des odes composées après les guerres (voir infra partie 3).

Les poèmes qui ne célèbrent pas des vainqueurs thébains présentent pour l'examen de notre question un avantage certain : Thèbes n'y figure pas à titre d'élément obligé. Par conséquent, une mention de Thèbes ou de la matière de Thèbes sera davantage susceptible d'être considérée comme relevant d'une stratégie du poète.

Le corpus des poèmes concernés, dans l'ordre chronologique communément admis, est le suivant ${ }^{28}$ :

- huitième et cinquième Isthmiques (-478);

- deuxième et troisième Olympiques, première Néméenne (-476) ;

- troisième Néméenne, deuxième Pythique (-475);

- dixième Olympique, troisième et neuvième Pythiques, neuvième Néméenne (-474) ;

- quatrième Néméenne (-473) ;

- sixième Olympique (-468) ;

- neuvième Olympique (-466) ;

- septième et treizième Olympiques (-464) ;

- quatrième Pythique (-462) ;

- huitième Néméenne (-459) ;

- huitième Pythique (-446) ;

- dixième Néméenne (-444 ?).

\section{$-478-$ Isthmique 8}

En l'honneur du jeune Cléandros, un Éginète vainqueur au pancrace, Pindare compose une ode qui contient de claires allusions aux événements guerriers dont les Grecs viennent de sortir vainqueurs, et pourtant il ne cache pas sa préoccupation (I., 8, 5-11). Comme pour attaquer le thème de front, il claironne le nom de Thèbes dès la première antistrophe $(I ., 8,15 a)$, mais il va profiter de ce que Thèbes et Égine sont, au niveau de leurs mythes fondateurs, des "sœurs (jumelles) ${ }^{29}$ ", toutes deux issues du fleuve Asôpos, toutes deux aimées de Zeus (I., 8, 16a-23). Elles donneront leurs noms aux deux 
cités : ainsi le lieu d'origine du poète et le lieu d'origine du vainqueur célébré se trouvent liés dans le passé le plus lointain. Solidarité des origines qui permet de faire face en bonne compagnie aux reproches éventuels, d'autant plus légitimement qu'Égine avait dû encourir les mêmes reproches que Thèbes pour s'être naguère soumise à Darius (Hérodote, 6, 49), même si elle avait ensuite changé de camp (Hérodote, 7, 203) ${ }^{30}$. La suite est quasiment prévisible: on continuera de puiser dans le passé lointain (toujours la main de Zeus...) et l'on déploiera largement la geste d'Achille depuis ses origines $^{31}$. Ainsi, on rappellera aux auditoires panhelléniques des hauts faits connus de tous: Achille est certes convoqué pour mettre en évidence l'énergie puisée dans le passé d'Égine et qui vient de se manifester une fois encore, au présent, dans la victoire de l'Éginète Cléandros ; cependant, du même coup, Achille parlera pour Égine. Dans son ombre, on devine un plaidoyer «jumeau » pour Thèbes, cité jumelle et cité du poète. L'heure de ce nouveau plaidoyer jumeau viendra, un peu plus tard et avec la même stratégie, dans l'évocation d'Héraclès héros panhellénique, comme Achille, et cependant thébain : ce sera la quatrième Isthmique.

Une maxime l'avait annoncé dans les premiers vers: le temps se joue de nous et son cheminement est sinueux (I., 8, 14-15), mais « on peut y remédier ». Ici, le remède proposé est la mise en évidence d'un passé où s'est joué le destin de l'ensemble des Grecs, un destin dont Achille a tenu les clefs. Une maxime implicite parle donc pour Égine comme pour Thèbes, cité jumelle, cité du poète : les fautes du passé relèvent des sinuosités du temps dans son écoulement imprévisible. Le remède est à chercher dans les valeurs communes qui, elles, ne changent pas.

Ce poème réunit deux stratégies que l'on peut observer à l'œuvre lorsqu'il s'agit pour Pindare d'évoquer Thèbes : une stratégie directe, qui passe dans ce cas par l'énoncé du nom lui-même de Thèbes sur le «fronton» du texte, et une stratégie qu'on pourrait qualifier d'«oblique", sous la forme d'un plaidoyer qui ne dit pas son nom, en l'occurrence l'éloge d'Égine, un éloge dans l'ombre duquel se profile une argumentation propre également à défendre Thèbes.

\section{$-478 ?-$ Isthmique 5}

Datée généralement de -478, destinée à célébrer la victoire au pancrace de l'Éginète Phylacidas, la cinquième Isthmique retient avant tout l'attention par son évocation de la bataille de Salamine. L'heure n'est plus, semble-t-il, au silence pudique observé au sujet de Salamine dans la huitième Isthmique. On peut supposer que l'évocation de cette bataille constituait désormais une sorte d'obligation du poète en la circonstance : le destinataire de l'ode vient d'Égine et les Éginètes ont joué un rôle de premier plan lors de la bataille de Salamine $(I ., 5,43-50)^{32}$. On doit se demander cependant si Pindare ne retourne pas cette obligation en opportunité de plaider en sous-main la cause de Thèbes $^{33}$. Thèbes est nommée dans la deuxième antistrophe $(I ., 5,32)$ et le contexte dans lequel ce nom surgit n'est pas indifférent. C'est une véritable ronde de héros qui est évoquée pour illustrer l'affirmation que les exploits sont récompensés par des chants accompagnés d'instruments de musique : héros étoliens, thébains, argiens, spartiates $(I ., 5,28-33)$ précèdent les éginètes. Les héros éginètes sont à leur tour rattachés aux deux grands courants de l'épopée : la matière de Thèbes dans sa connexion avec la matière de Troie apparaît au travers de la première expédition contre Troie sous la conduite d'Héraclès, héros thébain par excellence. Elle offre un palier vers la seconde 
expédition de Troie sous la conduite des Atrides (I.,5, 35-38). Tout semble bien concourir à conforter l'image de Thèbes comme partie prenante d'une culture commune et tenant son rang parmi les cités grecques.

En outre, la mention explicite des instruments de musique, phorminx, aulos (I., 5, 27-28), pourrait faire résonner comme une harmonique la gloire de Thèbes, cité dont le récit des origines implique la musique de la phorminx d'Amphion, cité qui revendique la paternité de l'aulète Clonas, cité connue pour ses exécutants et son école de musique ${ }^{34}$.

\section{-476 - Olympique 2}

Pour Théron d'Agrigente, Pindare compose la deuxième Olympique, devenue célèbre par les spéculations qu'elle reflète sur l'au-delà de la vie. Théron est le prince d'une cité glorieuse, qui tient tête à Carthage, qui se lance dans un programme de constructions rendu possible par sa participation à la victoire d'Himère (-480). Or, l'ode qui lui est adressée à l'occasion de sa victoire à la course des chars à Olympie résonne avant tout du passé thébain. Il existe un lien pour légitimer ce choix: Théron fait remonter sa lignée à Thersandre (schol. ad 0., 2, 70e), né de Polynice et de la princesse Argeia (schol. ad $0 ., 2,76 \mathrm{~d}$ ) et se rattache ainsi aux Adrastides. L'intrusion de la matière de Thèbes est donc moins inattendue qu'on ne pourrait le penser à première vue, mais elle va faire l'objet d'une utilisation raffinée.

Une affirmation frappe tout d'abord : on ne peut abolir le passé, mais un sort favorable peut le faire oublier $(0 ., 2,15-22)^{35}$. Vérité générale, certes, mais qui, venue d'un poète thébain, fait penser aux reproches que Thèbes encourait alors. C'est dans le passé de Thèbes que Pindare va chercher l'illustration: les filles de Cadmos ont beaucoup souffert, mais elles jouissent désormais d'un bonheur perpétuel (0., 2, 22-30). Sémélé, aimée de Zeus et mère de Dionysos vit parmi les habitants de l'olympe, Inô jouit d'une vie « impérissable » (ớ $\varphi$ iı ov) dans les eaux, parmi les filles de Nérée. L'ode se propose de donner un sens à la victoire olympique de Théron, et l'on comprend d'abord que les difficultés rencontrées par Théron vont déboucher sur un bonheur permanent, bonheur qu'annonce la victoire olympique qu'il vient de remporter. Mais, en l'occurrence, on peut y regarder de plus près et se poser la question d'un autre niveau dans ce choix de la matière de Thèbes: ce passé thébain ne pourrait-il aussi préfigurer un destin de la cité, annoncer la fin de ses difficultés présentes?

Lorsque Pindare renverse la formule pour affirmer que la félicité peut être suivie de douleur (0., 2, 35-37), c'est encore le passé thébain qui est évoqué, Laïos tué par đđipe, Étéocle et Polynice, Thersandre enfin, fils de Polynice, « racine » lointaine de la famille des Emménides (0., 2, 38-47) et donc de Théron lui-même ${ }^{36}$. En d'autres termes, la " douleur » envoyée par la destinée $(0 ., 2,35-37)$ est interprétée comme un nouveau terreau sur lequel va finalement prospérer le succès ${ }^{37}$.

Le degré suprême de ce succès est constitué par l'accès à l'île des Bienheureux (0., 2, 68-83 ${ }^{38}$. Une fois encore, Pindare convoque le passé lointain de Thèbes. Trois Bienheureux sont nommément cités : Pélée, Cadmos, Achille. Jusqu'à ce point du texte, on se trouvait renvoyé à des références purement thébaines. Ici, de manière complémentaire, on retrouve le besoin d'insérer le passé thébain dans un passé conçu plus largement comme celui de l'ensemble des Grecs: les noms de Pélée et d'Achille font surgir la matière de Troie. En outre, le parallèle entre Thèbes et Troie fonctionne ici comme une annonce faite à Théron. En effet, si Pélée, par l'intervention de Thétis, se 
trouve dans l'île des Bienheureux avec son illustre fils, alors que Cadmos n'a pas de descendant à ses côtés, on peut en conclure que la place est libre pour Théron, Pindare jouant pour lui le rôle que Thétis a joué pour Achille ${ }^{39}$.

Ainsi Théron, par le rattachement de la famille des Emménides au passé thébain, offre grâce à sa victoire olympique un présage favorable tant pour Agrigente que pour Thèbes, c'est une sorte de «Thébain d'honneur » que le sort vient de favoriser ${ }^{40}$. Et cela dans un contexte que le poète organise de manière telle qu'il présente des lueurs d'espoir pour l'image de Thèbes.

\section{-476 - Olympique 3}

La troisième Olympique résulte d'une commande du même Théron pour célébrer la même victoire. On considère généralement que le lieu d'exécution différait: la deuxième Olympique était probablement destinée à une exécution au palais de Théron, la troisième sans doute à une exécution lors de la fête agrigentine des Théoxénies (schol. ad 0., 3, 1a) $)^{41}$.

40 L'image de Thèbes est mise en jeu au travers de la figure d'Héraclès, incontestable héros thébain et panhellénique ${ }^{42}$. S'agissant des jeux olympiques, Pindare choisit de rappeler comment Héraclès rapporta de chez les Hyperboréens les oliviers destinés tout à la fois à ombrager le site du sanctuaire et à donner les branches dont on fait les couronnes des vainqueurs aux jeux $(0 ., 3,11-35)$. Donc, pourrait-on dire, rien de plus qu'une occurrence classique de l'énoncé du mythe fondateur, comparable à l'évocation de Pélops dans la première Olympique. Deux traits, cependant, résonnent comme une allusion cryptée à l'image de Thèbes. C'est tout d'abord Héraclès montré en compagnie des Dioscures (0., 3, 33-38), une forme d'insistance sur l'appartenance du héros thébain à l'ensemble des héros grecs. C'est ensuite, la conjonction étant établie, la répétition des noms des Dioscures et d'Héraclès dans des positions choisies: les Dioscures ont favorisé Théron en récompense des honneurs qu'il leur accorde à l'occasion de la fête des Théoxénies, lors de laquelle on exécute la troisième Olympique, et c'est l'occasion de les nommer une nouvelle fois $(0 ., 3,38-41)$; quant à Héraclès, la proclamation finale de son nom, qui tire à sa suite la gloire de Thèbes, est réservée pour le sommet de l'ode : les derniers mots joignent la gloire d'Héraclès à celle de Théron ${ }^{43}$ grâce à l'évocation d'une extrémité du monde ${ }^{44}$, celle qu'atteint la gloire de Théron, à savoir les « colonnes d'Héraclès » $(0 ., 3,43-45)^{45}$.

\section{-476 ? - Néméenne 1}

41 L'un des mythes fondateurs des jeux de Némée est constitué par un exploit d'Héraclès : sa victoire sur le lion de Némée. On peut donc s'attendre à une présence du héros thébain dans les épinicies composées à l'occasion des jeux néméens. Cependant, quelques traits particuliers pourraient bien aller plus loin qu'une simple mention obligée.

Composée pour une victoire de Chromios d'Etna aux jeux de Némée, la première Néméenne, dans sa partie mythique, concentre l'attention de l'auditeur sur la naissance d'Héraclès (N., 1, 33-72). L'intention d'illustrer l'image de Thèbes au travers de son héros le plus panhellénique tient justement au choix de cet épisode, celui de sa victoire sur les serpents envoyés par Héra, du tumulte qui en résulte au palais d'Amphitryon, 
bref, d'un épisode qui se déroule entièrement à Thèbes ${ }^{46}$. Même l'évocation finale des exploits d'Héraclès ne nous fait pas quitter Thèbes: elle est mise dans la bouche de Tirésias, appelé par Amphitryon sur les lieux du prodige (N., 1, 60-72). Un détail mérite une attention particulière : la manière dont Pindare introduit Héraclès dans le tissu du poème. En effet, ce qu'il en dira n'entretient aucun rapport évident avec la victoire célébrée. Pindare rattache le choix d'Héraclès à l'énoncé de valeurs données pour communes: diversité des talents et importance des dons naturels $(N ., 1,25)$, rôles complémentaires de la force et de l'intelligence (N., 1, 26-28), bon usage de la richesse (N., 1, 31-32), solidarité des espoirs humains (N., 1, 32-33). Le poète (ou le chœur qui le représente ${ }^{47}$ ) l'affirme avec un « moi » placé en évidence avant le nom d'Héraclès ( $N ., 1$, 33), voilà qui semble conduire tout naturellement à l'évocation du célèbre Thébain devenu Olympien, voilà qui doit répondre à qui s'étonnerait de voir la naissance d'Héraclès, l'épisode le plus thébain de la geste du héros, former le corps d'un poème destiné à un chef sicilien d'une cité nouvellement fondée. En fait, avant même que le nom d'Héraclès ait été prononcé, l'attention est entraînée dans une série d'énoncés propre à créer une sorte de climat consensuel, un climat dans lequel on se sent irrésistiblement poussé à faire confiance au poète. Un poète qui va glorifier Thèbes au travers de la figure d'Héraclès.

\section{-475 ? - Néméenne 3}

Dans cette ode composée en l'honneur d'une victoire d'Aristocléidès d'Égine, une nouvelle apparition d'Héraclès est rendue d'autant plus visible qu'elle est présentée comme une possible erreur : le poète se serait laissé aller à l'évocation d'Héraclès parce que la mention des colonnes d'Héraclès, limite du monde, l'aurait entraîné sur ce terrain alors qu'il voulait chanter la gloire des Éacides, héros tutélaires d'Égine, l'île du vainqueur célébré $(N ., 3,19-28)$. Ce glissement vers le héros thébain, habilement introduit par ce procédé dans la texture de l'ode, va rendre possible sa présence au côté de Télamon et d'Iolaos : en effet, la première prise de Troie, puis le combat contre les Amazones, mentionnés comme titres de gloire de l'Éacide Télamon, impliquent la présence d'Héraclès au point qu'il n'est même plus nécessaire de le nommer ( $N ., 3$, $35-40)^{48}$. C'est une sorte d'omniprésence discrète que le poète assure au héros thébain, en même temps qu'il souligne la solidarité des matières de Thèbes et de Troie sans avoir l'air d'y insister : dès la troisième triade ( $N ., 3,43$ sq.) l'attention se concentre sur Achille, le plus glorieux des Éacides, de sang divin comme Héraclès, et dont les exploits d'enfant (chasser lions et sangliers, N., 3, 44-47) rappellent étrangement ceux d'Héraclès. Comme si, sous couvert de glorification des héros éginètes, on assistait à une forme dissimulée de promotion du grand héros de Thèbes.

\section{$-475 ?-$ Pythique 2}

Classée dans les odes pythiques, cette épinicie célèbre une victoire de Hiéron de Syracuse lors de jeux dont la localisation fait problème $e^{49}$. La date semble être $-475^{50}$. Au troisième vers, le poète prononce le nom de Thèbes, «l'opulente Thèbes " ( $\lambda_{1} \pi \alpha \rho \tilde{\alpha} v$

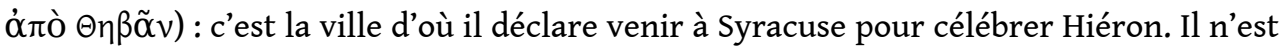
pas certain que cela signifie davantage que le simple envoi du poème de Thèbes en Sicile: le thème de l'hospitalité accordée au poète est comme on sait l'une des conventions du genre pour décrire les rapports du commanditaire et du poète ${ }^{51}$. La 
mention de Thèbes pourrait, elle aussi, être mise sur le compte d'une convention et n'avoir d'autre signification que d'indiquer la patrie de Pindare ${ }^{52}$. On est d'autant plus enclin à le penser que rien de thébain ne surgit dans le corps de l'ode. La partie mythique emprunte son sujet à la mésaventure d'Ixion. Il y a cependant une mise en accusation des calomnies (P., 2, 75-77), nuisibles à qui les écoute autant qu'à leur victime $^{53}$. Il est vrai que le contexte est celui de la cour d'un tyran sicilien et que l'image de Pindare surnageant comme le liège, qui ne plonge pas dans l'eau (P., 2, 80-81), ainsi que sa profession de foi en faveur de la franchise (P., 2, 86-88), pourraient se référer avant tout à des circonstances particulières à la Syracuse de l'époque, circonstances qu'au demeurant nous connaissons fort mal ${ }^{54}$.

\section{$-474 ?-$ Olympique 10}

Le cas de la dixième Olympique, écrite pour un vainqueur Locrien, présente une analogie avec celui de la deuxième Pythique: on y trouve une mention de Thèbes au travers de celle de Dircé $(0 ., 10,85)$ comme lieu d'où vient le chant qui célèbre le vainqueur. Cependant, à la différence du ton proclamatoire qui marque la mention de Thèbes au début de la deuxième Pythique, nous avons ici une allusion d'apparence plus discrète : c'est à la fin de l'ode que Pindare évoque sa provenance thébaine; ses chants sont venus, tardivement, « d'auprès de la célèbre Dircé » $(0 ., 10,84-85)$, la source désignant ici la ville de Thèbes comme le notent les scholiastes ${ }^{55}$. On ne saurait donc exclure une tentative de faire apparaitre Thèbes comme désirable : un chant très attendu, après un long délai, est finalement venu de là.

Par-delà la ressemblance qu'on peut noter ainsi entre la deuxième Pythique et la dixième Olympique dans la manière d'en user avec le nom de Thèbes ou de Dircé, il existe une différence de taille: si la deuxième Pythique ne contient pas d'éléments thébains, la dixième olympique donne à Pindare l'occasion d'exploiter la gloire panhellénique d'Héraclès. C'est une thématique à l'ombre de laquelle on devine de manière constante l'affirmation du rôle important de Thèbes dans le monde grec, mais les jeux olympiques sont évidemment l'occasion privilégiée de rappeler parmi les bienfaits que l'on doit au héros celui que constitue la fondation des jeux olympiques eux-mêmes (0., 10, 24-59). Au sommet des bienfaits que l'on peut retirer de l'acte fondateur d'Héraclès se trouve le chant de célébration, un acte des Muses auquel un Thébain, Pindare lui-même, déclare hautement qu'il apporte une aide essentielle (0., 10, 91-105). Il est à remarquer cependant que l'entrée d'Héraclès dans le poème mentionne une dérobade de ce dernier devant Cycnos $(0 ., 10,15-16)$. On s'est plu à penser qu'il s'agirait là d'un parallèle avec une mésaventure du jeune Locrien dont on fête la victoire ${ }^{56}$. Sans doute, mais une maxime ancrée dans la mort d'Augias vaincu par Héraclès donne à réfléchir :

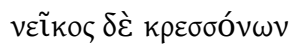

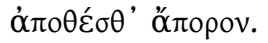

Il est impossible d'éviter le courroux des plus forts que soi.(0., 10, 39-40)

Il pourrait être également question, au-delà de l'immédiat, de situations que les Thébains ont connues. 


\section{-474 ? - Pythique 3}

Composée pour Hiéron de Syracuse, la troisième Pythique contient des thèmes qui semblent toucher profondément le destinataire et le poète ${ }^{57}$. Pour le destinataire, souffrant aux dires du scholiaste de calculs rénaux ${ }^{58}$, c'est le regret de l'absence de Chiron le guérisseur ( $P ., 3,1-7)$, l'évocation d'Asclépios, de ses parents, de sa naissance, de son action. Il en va de même pour le poète. Après avoir assuré une transition dans laquelle le destinataire et le poète ont partie liée ( $P ., 3,61-76$, nouvelle mention de Chiron et du rôle que le poète aurait pu jouer auprès d'Hiéron en gagnant le centaure à sa cause), Pindare va mettre en avant les sentiments qu'il éprouve lorsqu'il réfléchit au passé de Thèbes. Dans les chants qu'il entend «à sa porte» pendant la nuit en l'honneur de la Grande Mère et de Pan, il veut rappeler ce qu'enseignent les anciens : pour un bien, les immortels distribuent aux humains deux maux (P., 3, 77-83). La maxime s'applique à Hiéron, mais tout autant à Thèbes - et Pindare ne manque pas l'occasion de proclamer ici la formule «Thèbes aux sept portes» $(P ., 3,90-91)-$, comme le démontrent les destins de Pélée et de Cadmos. Leurs glorieux mariages avec des immortelles sont suivis de malheurs pour leur descendance immédiate ( $P ., 3$, 86-103). Les vents des hauteurs peuvent cependant changer de direction ( $P ., 3,104-105)$ et il importe de savoir se montrer au diapason des circonstances :

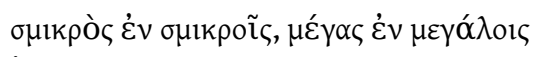

ह̌ $\sigma \sigma \circ \mu \alpha_{1}[. .$.

Humble dans l'humilité, je serai grand dans la grandeur (P., 3, 107-108).

Ainsi, revêtu du passé de Thèbes, Pindare se pose (lui ou son chœur) en parallèle de celui qui souffre et que les dieux viennent cependant de favoriser par la victoire pythique. «Les vents tournent » : si, pour Hiéron, une conjoncture pénible se change en bonheur et en gloire provenant des victoires qu'il a remportées aux jeux pythiques ( $P .$, 3, 73-74), le parallèle avec Pindare n'entraîne-t-il pas, dans l'ombre, l'annonce de vents meilleurs pour Thèbes?

\section{$-474-$ Pythique 9}

49 Écrite pour un Cyrénéen, Télésicrate, vainqueur à la course des hoplites, la neuvième Pythique débute par un rappel étendu du mythe de Cyrène aimée d'Apollon et transportée en Libye (P., 9, 1-70). Elle s'achève sur l'histoire libyenne de la fille d'Antée gagnée pour épouse, à la course, par Alexidamos à Irasa (P., 9, 103-125). Insérée entre ces deux séquences libyennes, Pindare situe au centre de son ode une partie thébaine (P., 9, 76-97). Elle débute par une maxime présentée comme couramment acceptée : il y a beaucoup à dire des grandes vertus $(P ., 9,76)$. Ainsi placée dans un climat d'approbation, «Thèbes aux sept portes » est alors nommée pour être prise à témoin d'épisodes légendaires rattachés à la figure illustre d'Héraclès $(P ., 9,79-89)$. Une polémique implicite semble parcourir ces vers: Pindare répond à une objection non formulée selon laquelle il devrait ne pas aborder cette matière. Sa réponse tient d'abord à ce qu'il faudrait être muet pour ne pas parler d'Héraclès $(P ., 9,87)^{59}$, elle tient ensuite à l'engagement personnel du poète qui affirme hautement la constance de son propos : il n'a cessé de louer Thèbes en divers lieux et l'on ne pourra le réduire au silence ( $P$., 9 , $90-92)$; il a toujours agi pour le bien public, ce que même ses ennemis devraient reconnaître $(P ., 9,93-94)$. Cette dernière considération permet un lien avec une 
nouvelle maxime, attribuée au "vieillard de la mer » : pour être juste, il faut savoir louer ses ennemis mêmes ( $P ., 9,94-96)$. Le segment thébain est particulièrement affirmatif, il implique le «moi » du poète (peut-être transféré au chœur) dans un contexte de contestation qui fait évidemment songer à l'image de Thèbes dans cette période délicate autant qu'aux difficultés rencontrées par Pindare lui-même auprès de ses concitoyens ${ }^{60}$. Le poète semble avoir redoublé de précautions en insérant ce segment entre deux maximes consensuelles d'abord, puis entre deux parties libyennes riches de narrations capables de mobiliser la mémoire de l'auditeur. Le climat polémique évoqué autour de Thèbes est ainsi habilement estompé pour laisser place dans la mémoire à la séduction des récits, mais sans qu'il ait fallu pour autant sacrifier la défense de l'honneur thébain.

\section{-474 ? - Néméenne 9}

Pour célébrer la victoire à Sicyone de Chromios d'Etna à la course de chars, Pindare commence par tabler sur le passé des jeux de Sicyone ${ }^{61}(N ., 9,8-12)$. Le rattachement des jeux sicyoniens à Argos permet une transition naturelle vers l'expédition des « Sept contre Thèbes ", qui fut menée à partir d'Argos (N., 9, 13-27). Ainsi la matière de Thèbes se trouve-t-elle mise au service de plusieurs objectifs : rappeler la gloire de la cité, rappeler dans le cas particulier la défaite de ses ennemis, offrir ainsi un précédent de bon augure à un vainqueur sicilien au moment où la menace carthaginoise pèse sur la Sicile $(N ., 9,28-30)^{62}$. La malveillance éventuelle à l'égard des Thébains se trouve ainsi combattue par un recours au passé thébain: Thèbes est profilée comme une cité victorieuse dont le bonheur légendaire constitue un gage d'avenir assuré pour des Grecs menacés. Des circonstances qui n'invitent pas à la dénigrer.

\section{-473 ? - Néméenne 4}

Composée pour un vainqueur éginète, la quatrième Néméenne fait une large place à l'image de Thèbes. La première occasion est offerte par le vainqueur lui-même: Timasarque d'Égine a remporté précédemment des couronnes aux jeux de Cléones, d'Athènes et de Thèbes ( $N ., 4,16-22)$. Placée de la sorte en troisième position, la proclamation du nom de Thèbes offre l'occasion d'une véritable salve : «ville aux sept portes", "tombeau glorieux d'Amphitryon", "Cadméens", "cour opulente d'Héraclès » (N., 4, 19-24). De plus, la passerelle vers les exploits d'Héraclès s'offre d'elle-même, sans qu'il faille s'éloigner par trop d'Égine, dès lors que Télamon accompagne Héraclès (N., 4, 25-32). C'est ici que Pindare place un segment polémique : il semble justement se défendre de critiques qui lui reprocheraient le choix de son sujet thébain, des gens « qui ne comprennent pas » ce qu'il dit (N., 4,31). Mais il annonce un triomphe et affirme sa confiance en son talent avant de poursuivre son chant en l'honneur des héros d'Égine ( $N ., 4,30-46)$. On se trouve devant une manière habile d'évoquer l'image de Thèbes au travers de son grand héros: imaginaire ou réel, le reproche de s'attarder sur une matière qui ne conviendrait pas à la célébration d'un Éginète attire justement l'attention sur la présence de Thèbes dans l'ode, et du même coup sur la claire intention du poète. Sa digression "théorique » lui permet d'insister sur le fait qu'il est le maitre du chant et que ce n'est pas le sujet qui le dirige. Autrement dit, si Thèbes est évoquée, c'est qu'il l'a bien voulu, et qu'il met son talent à son service. 
Le compagnonnage d'Héraclès et de Télamon est d'ailleurs propre à rappeler une caractéristique couramment exploitée par Pindare: la solidarité de la matière de Thèbes avec le passé du monde grec dans son ensemble en même temps que l'importance de cette matière. Importance telle que le poète offrira à son auditoire le luxe de l'évoquer sans la nommer, et cela, une nouvelle fois, dans un passage "théorique »: lorsque le poème risque de se prolonger indûment, Pindare utilise l'image du bateau qu'il faut ramener à sa bonne trajectoire pour éviter qu'il n'aille trop loin. Ce rappel à la juste mesure est exprimé par l'évocation d'une limite géographique : on ne peut aller plus avant que Gadès en direction du couchant (N., 4, 69-70). Ainsi, les " colonnes d'Héraclès » se profilent dans le texte sans qu'il soit même nécessaire de les nommer : un concept admis de tous est lié à Thèbes et l'auditeur en a la preuve au détour de cette remarque dont l'objectif affiché concerne la marche du poème.

\section{-468 - Olympique 6}

Pour célébrer Agésias de Syracuse, lieutenant de Hiéron, vainqueur à la course de chars attelés de mules, Pindare choisit un des mythes fondateurs du sanctuaire d'Olympie : la naissance du devin Iamos. Deux passages, toutefois, comme sertis dans ce monde olympique, sont en rapport direct avec Thèbes.

L'éloge que le vainqueur mérite, selon Pindare, peut être tiré d'un épisode de la guerre des Sept contre Thèbes: il s'agit de la formule dont se serait servi Adraste, chef de l'expédition, à Thèbes, pour déplorer la mort du guerrier et devin Amphiaraos :

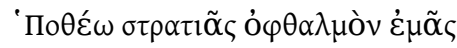

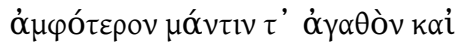

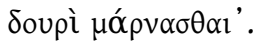

Il me faut l'œil de mon armée, tout à la fois bon devin et lancier redoutable à

la guerre. $(0 ., 6,16-17)$

La référence est précédée d'un passage gnomique sur les vertus qui doivent, pour être honorées, savoir affronter des dangers.

Vers la fin de l'ode, le nom de Thèbes résonne à nouveau, mais cette fois-ci au travers du nom de la nymphe Thébé, éponyme de la ville de Thèbes $(0 ., 6,84-85)$. Cette évocation est incluse dans un passage à la première personne du singulier. Pindare commence par se dire saisi d'une inspiration soudaine $(0 ., 6,82-83)$ : Stymphale, un lieu de cette Arcadie où se situe la naissance d'Iamos, offre un lien avec le monde du poète. C'est là qu'est née « la mère de la mère » du poète $(0 ., 6,84)$, en l'occurrence Métôpé mère de Thébé « mère » de Pindare (cf. I., 1, 1).

Ces deux touches thébaines pourraient se justifier simplement par le fait que l'auteur du poème est un Thébain, certes, mais on est en droit de se demander ce qui le pousse à l'affirmer, et surtout, peut-être, à l'affirmer de manière si habile : les deux touches sont en effet justifiées de manières très différentes. La première semble entraînée comme naturellement dans le courant du texte par une manière de dire qu'on peut faire communément remonter à l'épopée thébaine; elle fait suite à un passage de "sagesse des nations». La seconde est très visiblement introduite par l'expression d'un élan donné pour ressenti par le poète. Encadrant la longue évocation de la naissance d'Iamos et du privilège qui lui échoit à Olympie, la première allusion pourrait passer pour dissimulée dans les replis du récit, cependant que la deuxième est l'objet d'un geste 
démonstratif, accompagné d'une exhortation au chef du chœur invité à défendre l'honneur poétique des Béotiens (0., 6, 82-90), qui lui confère une grande visibilité.

À partir de ce moment, dans les odes qui vont suivre, on observe une sorte de césure ${ }^{63}$. Le nom de Thèbes continue d'apparaître, mais les contextes ne sont plus de même nature. On peut en retirer l'impression qu'il n'est désormais plus nécessaire de plaider la cause de Thèbes.

\section{-466 - Olympique 9}

Dans cette épinicie écrite en l'honneur d'un lutteur d'Oponte, Thèbes n'apparaît que de manière discrète et quasiment anecdotique. Héraclès se trouve mentionné à propos d'un sujet dont il ne faut pas traiter $(0 ., 9,29-41$, le combat d'Héraclès à Pylos contre trois dieux ${ }^{64}$. Le nom de la cité retentit dans le contexte de l'histoire ancienne d'Oponte: Thèbes est l'une des cités grecques qui, avec Argos, Pise, Égine ou l'Arcadie, envoyèrent des citoyens rejoindre à Oponte son héros fondateur et éponyme $(0 ., 9$, 67-70). Par ailleurs, sans même que le nom de Thèbes soit mentionné, Pindare prononce l'éloge de Lampromachos, vainqueur naguère aux jeux de l'Isthme, parent du vainqueur et "proxène » des Thébains à Oponte $(0 ., 9,83-85)$. Au chapitre qui serait celui de l'image de Thèbes, le poème donne l'impression très nette que la cité aurait en quelque sorte repris sa place traditionnelle.

\section{-464 - Olympique 7}

59 La même impression prévaut dans la célèbre septième Olympique, si belle que dans la patrie du vainqueur, à Rhodes, on la transcrivit en lettres d'or ${ }^{65}$ : le nom de Thèbes est mentionné dans une liste de lieux où Diagoras, le boxeur en l'honneur de qui l'épinicie fut composée, remporta des victoires (0., 7, 80-87). Rien de plus.

\section{-464 - Olympique 13}

$\mathrm{Au}$ cours de la même année, Pindare a célébré dans sa treizième Olympique un Corinthien, Xénophon, vainqueur au stade et au pentathle. Le nom de Thèbes est prononcé dans le même contexte que dans la septième Olympique: Xénophon a remporté une série de victoires parmi lesquelles figurent celles obtenues à Thèbes (0., 13, 106-113).

\section{$-462-$ Pythique 4}

61

Dans ce poème, le plus long des poèmes conservés de Pindare, le poète célèbre la victoire d'Arcésilas, roi de Cyrène, vainqueur à la course des chars à Delphes. On y trouve une évocation de la légende des Argonautes, liée au fait que la famille royale de Cyrène se considérait comme descendante de l'Argonaute Euphamos, fils de Poséidon. Thèbes, implicitement présente dans une mention de la « sagesse d'CEdipe » $(P ., 4,263)$, est nommément citée dans le dernier vers $(P ., 4,299)$ : c'est la conclusion du segment dans lequel Pindare fait allusion aux contacts personnels qu'il a établis avec Damophilos, Cyrénéen tombé en disgrâce et dont il prend la défense ${ }^{66}$. C'est dire que s'il est une image que le poète s'ingénie à corriger, ce n'est justement plus celle de Thèbes. 


\section{-459? - Néméenne 8} d'un passage gnomique, émet un vœu personnel : que ses concitoyens lui donnent leur approbation (N., 8, 38-39). À cette occasion, le nom de Thèbes n'est même pas prononcé. Est-ce à dire qu'il chercherait à le cacher ? On peut affirmer le contraire, car la matière de Thèbes est ouvertement convoquée en fin de poème : il y eut des chants de triomphe dans les temps anciens, avant même que ne surgisse "la dispute entre Adraste et les Cadméens » (N., 8, 50-51), ce qui, dans une ode pour un vainqueur aux jeux de Némée, évoque la fondation des jeux à l'occasion des funérailles d'Archémoros et, du même coup, un temps précédant la fondation des jeux de Némée ${ }^{67}$. Le contexte implique cependant que la guerre des Sept contre Thèbes sert principalement de pur repère chronologique.

\section{$-446-$ Pythique 8}

Par comparaison avec l'ensemble des odes postérieures à la "césure ", on se trouve avec la huitième Pythique devant un cas radicalement différent.

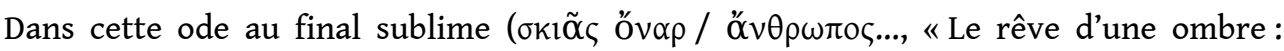
l'homme... ", P., 8, 95-96), écrite pour fêter la victoire pythique du lutteur éginète Aristoménès, on voit revenir en force la matière de Thèbes. S'agissant d'un vainqueur d'Égine, la parenté fréquemment soulignée de Thèbes et d'Égine peut expliquer le fait. Mais cela suffit-il ? Des allusions à l'expédition des Épigones occupent le segment mythique de l'ode $(P ., 8,39-56)^{68}$. À la faveur d'un parallèle portant sur la prophétie de succès futurs, Pindare applique au cas du vainqueur une déclaration d'Amphiaraos prononcée lors de la seconde expédition argienne contre Thèbes $(P ., 8,44-55)$. C'est donc de manière très visible que l'épopée thébaine vient occuper une place d'honneur.

Si l'on considère le contenu de la déclaration prêtée à Amphiaraos, on constate qu'il est question d'abord de la valeur qui se transmet de génération en génération, ce qui convient au parallèle établi avec la famille du vainqueur célébré dans cette épinicie. Mais les dires d'Amphiaraos vont plus loin, ils s'éloignent du simple éloge des qualités héritées de nature : ce qui conclut la prise de parole du devin, c'est le malheur qui va frapper Adraste en plein succès, " au milieu de sa troupe indemne » $(P ., 8,54)$, à savoir la perte de son fils. On se trouve devant un exemple spectaculaire qui doit donner à réfléchir sur les mutations des destins humains.

Or, la huitième Pythique s'achève justement par une déclaration devenue célèbre sur la fragilité de la condition humaine $(P ., 8,95-100)^{69}$. On est tenté de considérer que la place d'honneur faite au mythe thébain tient au fait qu'il constitue une sorte de préparation du segment gnomique final. Dans la matière de Thèbes, le poète trouve l'autorité qui justifie sa position finale, le destin changeant des adversaires de Thèbes, leur gloire, leurs chutes, leur renommée, permettent de prononcer les mots par lesquels, nouvel Amphiaraos, le poète va conclure son œuvre. Jusqu'à un certain point, la situation se renverse: ce n'est plus un segment gnomique qui donnerait du sens au segment thébain que le poète aurait appelé à la rescousse de l'image de Thèbes, c'est ici la matière de Thèbes qui offre au segment gnomique, sommet de l'ode, son fondement le plus assuré. 


\section{-444? - Néméenne 10}

La date de ce poème est l'objet de conjectures très diverses : la fourchette se situe entre -500 et -444 , c'est dire si l'on est dans l'incertitude. De fait, cette épinicie en l'honneur de l'Argien Théaios, vainqueur à la lutte, n'est pas une Néméenne, malgré son classement (tout comme la neuvième Néméenne, voir n. 61).

La première triade célèbre les gloires légendaires d'Argos, comme on peut s'y attendre lorsqu'il est question d'un vainqueur argien. Thèbes n'apparait que dans la marge des légendes argiennes : c'est une allusion à la guerre des Sept contre Thèbes (Amphiaraos l'Argien englouti par la terre: N., 10, 8-9), une mention d'Alcmène (aimée de Zeus comme Danaé : N., 10, 10-11), et l'évocation d'Amphitryon, explicitement donné comme argien, mais dont Zeus prit la figure pour engendrer Héraclès (N., 10, 15-18). On remarque à ce propos que la figure d'Héraclès est subrepticement présentée comme un titre de gloire d'Argos, toute allusion à Thèbes étant évitée lorsqu'il est question de lui dans l'épode de la première triade (N., 10, 13-18). Pour Pindare, au moment où il compose cette épinicie, et quel que soit ce moment, il semble bien que plaider la cause de Thèbes ne soit plus, ou pas encore, à l'ordre du jour.

\section{Observation finale à propos des poèmes antérieurs à la fin des guerres médiques}

69 Il est temps d'en venir à la vérification que nous permet l'examen des odes composées avant la fin des guerres médiques. On peut y constater, en effet, qu'il existe dans les épinicies de Pindare un traitement particulier de Thèbes, de son passé, de son image, après les guerres médiques: un survol des poèmes qui furent composés avant -480 , donc avant le moment où les Thébains durent affronter les reproches des Grecs, fait apparaître que la thématique thébaine n'y remplit pas la fonction qu'on peut lui voir après la bataille de Platées.

Sur les neuf épinicies dont la date de composition précède celle de la fin des guerres médiques $^{70}$, trois mentionnent des faits thébains: la dixième Pythique (-498), la septième Néméenne (-485 ?) et la sixième Isthmique (-480 ?).

\section{-498 - Pythique 10}

71 Dans ce poème de jeunesse, Pindare affiche clairement une intention d'inclure une dimension panhellénique à son épinicie. Le bonheur de Sparte et de la Thessalie, décliné en termes distincts et soigneusement distribués, tient à ce que l'un et l'autre lieu ont pour dirigeants des descendants d'Héraclès $(P ., 10,1-3)$. La descendance du héros thébain se trouve ainsi rattachée à la dynastie des Aleuades de Thessalie, donc de Thôrax, commanditaire de l'ode et par ailleurs allié des Perses ${ }^{71}$. On est très nettement en deçà d'une situation où l'image de Thèbes serait altérée par un contact avec les Perses. 


\section{-485 ? ${ }^{72}$ - Néméenne 7}

72 La septième Néméenne, composée pour l'Éginète Sôgénès vainqueur au pentathle des garçons, met en jeu une polémique qui avait surgi entre Pindare et certains Éginètes à propos du personnage de Néoptolème, fils d'Achille ${ }^{73}$. On suppose que la discussion avait été rude, car les Éginètes n'étaient sans doute pas prompts à pardonner des écarts perçus comme déplaisants à propos de la lignée des Éacides, une lignée héroïque profondément liée au passé de leur île. C'est dans ce contexte que Pindare est amené à évoquer sa cité de Thèbes, mais c'est pour se défendre à titre personnel et dire qu'il jouit de l'estime de ses concitoyens (N., 7, 64-69). Plus loin, dans la cinquième triade, Pindare invoque la protection d'Héraclès pour le vainqueur et son père $\left(N_{.}, 7,86-101\right)$ : Sôgénès habite une maison encadrée de sanctuaires d'Héraclès. Rien ne donne l'impression que l'on dépasse ici la simple fierté d'être citoyen de Thèbes et de pouvoir dire à l'honneur d'un vainqueur qu'il est un « bon voisin » (N., 7, 87-89) témoignant son respect au héros thébain Héraclès. L'essentiel, en fait, n'est justement pas là.

\section{$-480-$ Isthmique 6}

Dans la sixième Isthmique, composée en l'honneur de Phylacidas d'Égine, vainqueur au pancrace des garçons, deux passages sont en rapport direct avec Thèbes. À la fin de l'ode, faisant allusion à sa propre action de poète, Pindare déclare qu'il abreuvera le vainqueur de l'eau pure de Dircé, cette eau que les filles de Mnémosyne firent surgir près des portes des beaux remparts de Cadmos (I., 6, 74-75). À l'évidence, ces éléments thébains n'ont d'autre propos que d'indiquer par un détour que le poète est thébain.

Cependant, dans le corps du poème, on voit apparaître Héraclès en gloire (I., 6, 27-54). À travers lui, Pindare met en lumière les Éacides, comme on peut s'y attendre lorsqu'il est question de célébrer le succès d'un Éginète. L'épisode choisi nous indique une direction. Il est en effet question de la première guerre de Troie, celle qui vit Télamon se joindre à Héraclès. L'évocation se produit en deux temps: survol d'exploits accomplis en commun $(I ., 6,25-35)^{74}$, puis retour au point de départ, en l'occurrence à la scène d'Héraclès reçu chez Télamon lorsqu'il vint lui proposer de se joindre à l'expédition de Troie (I., 6, 35-56). Cette scène comporte une prière adressée à Zeus ainsi qu'une prophétie de la naissance d'Ajax (avec jeu de mots sur l'aigle qui est apparu après la prière : $\underline{\text { ai }}$ (tos-Aias), toutes deux prononcées par Héraclès ${ }^{75}$. Faut-il voir dans ce segment de l'épinicie une exhortation à l'alliance d'Égine et de Thèbes contre un ennemi situé sur l'autre rive de la mer? Ce serait un argument en faveur d'une datation à -480 plutôt qu'à -484 . Ce serait alors, et peut-être surtout, une indication pour la position de Pindare dans le conflit qui menace ${ }^{76}$, celle d'une solidarité de Thèbes avec d'autres cités grecques sur le modèle de celle qu'avait suscitée contre Troie le grand héros thébain. Mais, en tout état de cause, il ne serait pas question de défendre l'image de Thèbes.

Considérons en outre, toujours pour cette même période, deux odes dans lesquelles n'apparaît aucun élément thébain : la sixième Pythique, et la cinquième Néméenne. Sans qu'il y soit question de Thèbes, toutes deux présentent ce qu'on pourrait appeler des « occasions manquées » si on les rapproche des poèmes qui ont suivi la fin des guerres médiques. 


\section{-490 - Pythique 6} passé de Thèbes et l'usage qu'en fait Pindare. Dans la sixième Pythique, composée en l'honneur de l'Agrigentin Xénocrate, frère de Théron qui allait devenir le tyran d'Agrigente, Pindare ne manque pas d'évoquer les «prospères Emménides » (5), mais il ne ressent aucun besoin de les rattacher à Thèbes.

\section{-483? - Néméenne 5}

On se souvient également de quelle manière Pindare se sert de la proximité d'Égine et de Thèbes dans des odes destinées à des vainqueurs éginètes et composées après la fin des guerres médiques. Si l'on compare cette manière de procéder avec ce qu'on trouve dans la cinquième Néméenne, composée en l'honneur de Pytheas d'Égine, vainqueur au pancrace « des imberbes ", on est frappé par la totale absence de toute référence à une solidarité d'Égine et de Thèbes.

On le constate: la manière dont Thèbes et le passé thébain se manifestent dans les épinicies de Pindare antérieures à la fin des guerres médiques est en net contraste avec l'usage que le poète en fera après la fin de l'invasion de Xerxès. On se trouve ainsi conforté dans l'idée que l'image ternie de Thèbes devient alors une préoccupation qui détermine dans les épinicies pindariques, en contrepoint plus ou moins perceptible avec leurs sujets principaux, ce qu'on pourrait définir comme de véritables stratégies pour Thèbes.

\section{Conclusion}

Si l'on considère l'ensemble des stratégies qui pourraient chez Pindare concourir à une sorte de plaidoyer en faveur de Thèbes dans les odes composées après la fin des guerres médiques, on rappellera pour commencer qu'il convient d'écarter d'emblée l'utilisation en elles-mêmes des victoires obtenues par des concurrents thébains comme signalant la bienveillance des dieux. Il s'agit là d'une donnée constitutive des jeux organisés dans les sanctuaires. Ces victoires et leur utilisation ne sauraient par conséquent être mises au compte d'une stratégie particulière du poète.

Pour le reste, on observe à l'évidence une variété des procédés ainsi que des nuances conditionnées par les circonstances de production des poèmes. Cependant, on trouve également des constantes. Deux approches principales méritent d'être distinguées, celles qui nous sont apparues dès la huitième Isthmique (supra): le poète peut se prononcer ouvertement en faveur de Thèbes, alléguant éventuellement des motifs appropriés, ou choisir de procéder de manière oblique, en évoquant des événements ou des valeurs qui permettent de considérer Thèbes sous un jour favorable.

Dans la première catégorie, celle de l'affirmation frontale, on trouve bien évidemment la déclaration d'attachement à sa cité $(0 ., 6 ; P ., 3 ; I ., 1$ et 7), on trouve l'idée d'une solidarité qui, dans le passé déjà, unit Thèbes et d'autres cités grecques $(P ., 11 ; N ., 4$; I., 3-4 et 8). Comme on peut s'y attendre, le personnage d'Héraclès joue un rôle important dans ce contexte, en sa qualité de Thébain et de héros panhellénique tout à 
la fois $(0 ., 10 ; I ., 3-4 ; I ., 7 ; I ., 8)$. Une variante appuyée de cette procédure consiste à fixer l'attention sur ce qui peut unir Héraclès et la seule Thèbes $\left(N_{.}, 1\right)$.

La seconde catégorie, celle de la manière oblique, présente des cas plus nombreux et plus riches de variables. On peut y voir principalement les procédés suivants, parfois mis en œuvre simultanément :

- évoquer la solidarité de la matière de Thèbes avec divers éléments du passé commun des Grecs, dont la matière de Troie $\left(0 ., 2 ; 0 ., 3 ; P ., 11 ; I_{.,} 3 ; I ., 5 ; I ., 8\right)$;

- évoquer des destins marqués par des vicissitudes semblables à celles que Thèbes a traversées (0., 2; I., 3-4). Le passé thébain peut même être de bon augure pour d'autres cités (N., 9) ;

- évoquer des destins héroïques ressemblant à celui d'Héraclès (I., $\left.8 ; N_{\text {., }} 3\right)$.

On observe en outre ce que l'on pourrait appeler des procédures de composition :

- faire précéder ou accompagner un élément thébain de l'énoncé de valeurs consensuelles - notamment de maximes affirmant que l'erreur peut être suivie de succès - pour créer un climat d'approbation préalable (0., $6 ; P ., 3 ; P ., 9 ; N ., 1 ; I ., 3-4)$;

- attirer l'attention sur l'élément thébain en évoquant la possibilité qu'il soit hors de propos, voire provoqué par un élan inexplicable $(0 ., 6 ; N ., 3 ; N ., 4)$;

- inversement, utiliser la disposition des matières pour "cacher » l'élément thébain par la prépondérance d'autres sujets et leur position dans le texte $(P ., 9)$ ou pour faire attendre la mention de Thèbes $(0 ., 10)$.

Il est évident toutefois que la mise en évidence de telles constantes ne revient pas à démontrer qu'il existerait une application mécanique de quelques procédés. Il suffit de se reporter à chacun des poèmes concernés pour observer la variété des solutions mises en œuvre par Pindare à chaque fois qu'il entend introduire, ouvertement ou allusivement, une touche thébaine pour défendre l'image de Thèbes.

\section{BIBLIOGRAPHIE}

BOCKSBERGER Silvain, « À la gloire des Cléonymides. Les deux poèmes de Pindare pour Mélissos de Thèbes : la $3^{\mathrm{e}}$ et la $4^{\mathrm{e}}$ Isthmiques ", QUCC, 1, 2009, p. 95-120.

BOWRA Cecil Maurice, Pindar, Oxford, University Press, 1964.

BRASWELL Bruce Karl, A Commentary on Pindar Nemean One, Fribourg, University Press Fribourg Switzerland, 1992.

BRASWELL Bruce Karl, A Commentary on Pindar Nemean Nine, Berlin, Walter de Gruyter, 1998.

BURNETT Ann Pippin, Pindar's Songs for Young Athletes of Aigina, Oxford, Oxford University Press, 2005.

BURTON Reginald William Butler, Pindar's Pythian Odes. Essays in Interpretation, Oxford, Oxford University Press, 1962.

BURY John Bagnell, The Nemean Odes of Pindar, Londres-New York, Macmillan and Co., 1890.

BURY John Bagnell, The Isthmian Odes of Pindar, Londres-New York, Macmillan and Co., 1892. 
CAREY Christopher, A Commentary on Five Odes of Pindar, New York, Arno Press Inc., 1981.

DAUDE Cécile, DAVID Sylvie, FRATZOFF Michel \& MUCKENSTURM-POULLE Claire, Scholies à Pindare, vol. I : Vies de Pindare et scholies à la première Olympique. « Un chemin de parole » (0.1.110), Besançon, Presses universitaires de Franche-Comté, 2013.

DRACHMANN Anders Bjorn, Scholia vetera in Pindari carmina, vol. 1 : Scholia in Olympionicas, Leipzig, Teubner, 1903.

DRACHMANN Anders Bjorn, Scholia vetera in Pindari carmina, vol. 2 : Scholia in Pythionicas, Leipzig, Teubner, 1910.

DRACHMANN Anders Bjorn, Scholia vetera in Pindari carmina, vol. 3 : Scholia in Nemeonicas et Isthmionicas. Epimetrum. Indices, Leipzig, Teubner, 1927.

EVEN Jean-Charles, «L'attitude de Pindare pendant les guerres médiques », Les Études classiques, XXVI, 1958, p. 41-49.

FARNELL LEWIS Richard, The Works of Pindar, Translated with Literary and Critical Commentary, vol. 2 : Critical Commentary, Londres, Hakkert, 1932.

FEARN David, Pindar's Eyes. Visual and Material Culture in Epynician Poetry, Oxford, Oxford University Press, 2017.

FINGLASS Patrick, Pindar Pythian Eleven (edited with Introduction, Translation and Commentary), Cambridge, Cambridge University Press, 2007.

GENTILI Bruno, ANGELI BERNARDINI Paola, CINGANO Ettore \& GIANNINI Pietro, Pindaro, Le Pitiche (Introduzione, testo critico e traduzione), Milan, Mondadori, 1995.

GENTILI Bruno, CATENACCI Carmine, GIANNINI Pietro \& LOMIENTO Liana, Pindaro, Le Olimpiche, Milan, Mondadori, 2013.

GIANNINI Pietro, « Le antiche tradizioni tebane negli epinici di Pindaro », dans P. Angeli Bernardini, Presenza e funzione della città di Tebe nella cultura greca (Atti del Convegno internazionale, Urbino, 7-9 juillet 1997), Pise-Rome, Istituti editoriali e poligrafici, 2000, p. $163-178$.

GILDERSLEEVE Basil, Pindar. The Olympian and Pythian Odes, New York, Cincinnati, Chicago, American Book Company, 1885.

HENRY Ben, Pindar's Nemeans. A Selection, Munich-Leipzig, Saur, 2005.

HUMMEL Pascale, L'épithète pindarique. Étude historique et philologique, Bern, Peter Lang, 1999.

HURST André, « Observations sur la deuxième Olympique de Pindare », Živa Antika, 31, 1981, p. 121-133.

HURST André, « Aspects du temps chez Pindare », dans Pindare, Vandœuvres-Genève, Entretiens Hardt, 1985, p. 155-197.

HURST André, « Der Dichter spricht : Pindare entre les lignes dans la quatrième ode pythique », dans C. Musuteanu, M. Barbulescu \& D. Benea (éd.), Corona Laurea. Studii in onoarea Luciei Teposu Marinescu, Bucarest, Muzeul de Istorie a Romaniei, 2005, p. 241-245.

KAMBYLIS Athanasios, Eustathios von Thessalonike, Prooimion zum Pindarkommentar. Einleitung, kritischer Text, Indices, Göttingen, Vandenhoeck \& Ruprecht, 1991.

LEFKOWITZ Mary, First-Person Fictions: Pindar's Poetic 'I', Oxford, Clarendon Press, 1991. 
LLOYD-JONES Hugh, « Pindar and the After-Life », dans Pindare, Vandœuvres-Genève, Entretiens Hardt, 1985, p. 245-283.

MÉAUTIS Georges, Pindare le Dorien, Neuchâtel, éditions de la Baconnière, 1962.

MULLEN William, Choreia, Pindar and Dance, Princeton, Princeton University Press, 1982.

OLIVIERI Oretta, Miti e culti tebani nella poesia di Pindaro, Pise-Rome, Fabrizio Serra, 2011.

PRIVITERA Aurelio, Pindaro, Le Istmiche, Milan, Mondadori, 2001.

PUECH Aimé, Pindare, t. I : Olympiques, Paris, Les Belles Lettres, 1931.

PUECH Aimé, Pindare, t. II : Pythiques, Paris, Les Belles Lettres, $1955^{3}$.

PUECH Aimé, Pindare, t. III : Néméennes, Paris, Les Belles Lettres, 1923.

PUECH Aimé, Pindare, t. IV, Isthmiques et fragments, Paris, Les Belles Lettres, 1961.

RADT Stefan Lorenz, Pindars zweiter und sechster Paian (Text, Scholien und Kommentar), Amsterdam, Hakkert, 1958.

ROESCH Paul, «L'aulos et les aulètes en Béotie », dans BOIOTIKA (Vorträge vom 5. Internationalen Böotien-Kolloquium), Munich, Maris, 1989, p. 203-214.

RUTHERFORD Ian, Pindar's Peans, Oxford, Oxford University Press, 2001.

SCHACHTER Albert, Cults of Boiotia, vol. 2, BICS Bulletin Supplement 38.2, Londres, Institute of Classical Studies, 1986.

SCHACHTER Albert, Boiotia in Antiquity. Selected Papers, Cambridge, Cambridge University Press, 2016.

SCHUBERT Paul, « La seconde entrevue de Pélias et Jason dans la $4^{\mathrm{e}}$ Pythique de Pindare : essai d'interprétation ", Antiquité classique, 73, 2004, p. 15-24.

SEVIERI Roberta, « Rimozione collettiva e riabilitazione individuale: Tebe dopo le guerre persiane nella prima Istmica di Pindaro », QUCC, 62 (2), 1999, pp. 35-48.

SEVIERI Roberta, «Cantare la città: tempo mitico e spazio urbano nell'Istmica 7 di Pindaro per Strepsiade di Tebe », dans P. Angeli Bernardini (dir.), Presenza e funzione della città di Tebe nella cultura greca (Atti del Convegno internazionale, Urbino, 7-9 juillet 1997), Pise-Rome, Istituti editoriali e poligrafici, 2000, pp. 177-190.

SNELL Bruno \& MAEHLER Herwig (éd.), Pindarus, Pars I: Epinicia, Leipzig, Teubner, $1971^{5}$.

WILAMOWITZ Ulrich, « Pindars siebentes Nemeisches Gedicht », Sitzungsberichte der KöniglichPreussischen Akademie der Wissenschaften, philosophisch-historische Klasse, XV, 1958, p. 328-352.

WILAMOWITZ Ulrich, Pindaros, Berlin, Weidmannsche Buchhandlung, 1922.

\section{NOTES}

1. Pour les événements de Thèbes, cf. Hérodote, 9, 86-88. Pour la situation de Pindare dans ce contexte, cf. e.g. Bury (1892, 133-134), Wilamowitz (1922, 184-195), Bowra (1964, 110-117). Bowra considère la situation comme clarifiée pour Pindare à partir du moment où sa renommée est telle qu'il n'a plus à pâtir du fait que Thèbes et Delphes avaient été du côté des Perses (pour lui, dès -479). Méautis $(1962,252-280)$ considère l'image de Thèbes chez Pindare, mais en se limitant aux quatre épinicies qui célèbrent des vainqueurs thébains. Les «Vies" antiques ne font pas mention d'une difficulté de la position de Pindare après le mauvais choix de Thèbes lors de la 
seconde invasion perse. Cf. Scholies, vol. I (1903), 1-11, Daude et al. (2013, 47-173), pas davantage que l'article qui lui est consacré dans la Souda. Rappelons que selon Polybe $(4,31)$ Pindare aurait approuvé et encouragé les Thébains dans leur position favorable à l'envahisseur. Wilamowitz (1922, 193-195), pour sa part, pense discerner une neutralité de Pindare dans le conflit.

2. Pour la datation des poèmes on se réfère à l'édition de Snell-Maehler $\left(1971^{5}\right)$.

3. Un cas extrême pourrait être celui d'Alcibiade lorsqu'il passe du côté de Sparte au moment de l'expédition de Sicile. Thucydide lui prête des propos selon lesquels, même en désaccord avec sa cité, il agirait par patriotisme (Thucydide, 6, 92).

4. Une cause que l'on peut définir selon les termes célèbres d'Hérodote $(8,144,2$ : parenté de sang, communauté de langue, communauté de rites religieux, parenté des styles de vie).

5. Pour un traitement d'ensemble du passé légendaire de Thèbes chez Pindare, on se reportera à Giannini (2000) et Olivieri (2011).

6. Thucydide, 2, 71-74 montre que lors du siège de Platée, le souvenir des guerres médiques était bien présent.

7. Pour son éloge d'Athènes, Pindare reçoit 10000 drachmes de la part des Athéniens (Isocrate, 15, 166). Il est mis à l'amende par les Thébains et les Athéniens acquittent l'amende pour lui. Cf. Daude et al. (2005, 60, 63 et 83, n. 48). Pour le témoignage d'Eustathe, cf. Kambylis (1991, 25, § 28).

8. Sur cette question, on consultera Mullen (1982). Dans la mesure où chorégraphie et arts visuels sont en contact, cf. Fearn (2017).

9. L'analyse de la métrique et des structures strophiques constitue ce qui nous reste de moins spéculatif. Il arrive en outre que le mode musical de l'ode soit indiqué (e.g. le mode lydien dans 0., 14, 17 ou dans N., 4,45), voire le mode et des instruments de musique (e.g. I., 4, 44-45). Cependant, il tombe sous le sens que, par exemple, les indications «sol majeur ", "cordes » et «vv- vv- vv- vv-», pour correctes qu'elles soient, ne donneraient qu'une bien faible idée du début du troisième concerto brandebourgeois de J. S. Bach.

10. Bury $(1892,167)$ opte pour deux poèmes distincts : «If there was originally a single ode, it is hard to see why it would come into the mind of anyone to separate the first metrical system from the rest. " Un argument répercuté parmi d'autres chez Privitera (20014, 43-44). Wilamowitz soutient le contraire (voir n. 11)

11. Farnell $(1932,348)$ démontre de manière convaincante que l'expression « enfants des dieux » n'est pas équivalente à "dieux", ce qui permet ici de penser à l'illustre Thébain Héraclès. Wilamowitz $(1922,335)$ tire argument de ce vers pour défendre l'unité des deux poèmes : aucun poème ne pouvait s'achever sur ces mots selon lui, en discuter serait du temps perdu : «Ich halte es für überflüssig, dabei zu verweilen, dass mit diesem Missklang kein Lied schliessen kann. »

12. Malgré les doutes qu'on a pu avoir à ce sujet, c'est l'opinion généralement admise. Cf. e.g. Privitera $\left(2001^{4}, 175\right)$. «L'absence d'indication précise permet de supposer que Pindare ne tient nullement à dévoiler un nom qui flétrit la réputation de Thèbes et évoque pour elle un passé chargé. » (Even, 1958, 46)

13. On a également supposé qu'ils avaient été exécutés après la défaite des Perses (Bocksberger, 2009, 98, 100-102).

14. Wilamowitz $(1922,339)$ observe que Pindare peut ici compter sur son public : il n'a pas même besoin d'évoquer le motif pour lequel Ajax encourt des reproches.

15. Pour une interprétation limitant la portée de l'exemple à la famille du vainqueur, cf. Bocksberger (2009, 107-108).

16. L'évocation d'un Héraclès de petite taille a surpris certains commentateurs (e.g. Farnell, 1932, 354). C'est un trait qui montre l'ancrage de Pindare dans les traditions thébaines : on connaît à Thèbes et à Thespies des représentations d'Héraclès de petite taille, cf. Schachter $(1986,34)$. 
17. On a bien évidemment spéculé sur les rapports de Pindare et d'Eschyle à ce propos, ainsi que sur les rapports éventuels de l'un et de l'autre avec l'Orestie de Stésichore et sur la place d'Oreste en Béotie ; e.g. Farnell (1932, 223-224), Gentili et al. (1995, 284-290), Finglass (2007, 11-17).

18. Thucydide, 3,62 et Puech $\left(1955^{3}, 156\right)$.

19. Un interprète aussi avisé que Burton se dit enclin à suivre le scholiaste (Burton, 1962, 63-64).

20. Cf. e.g. Gentili et al. (1995, 287-290), Finglass (2007, 109-110). En revanche : « Ganz äußerlich ist die Verbindung zum Mythos ", juge Wilamowitz (1922, 260).

21. Schachter $(2016,69)$ considère que les Thébains s'étaient déjà considérablement réhabilités (voire complètement réhabilités, cf. ibid., 105) en -474 lorsque Pindare compose la onzième Pythique et l'ensemble Isthmiques 3-4. On se gardera cependant de confondre des réalités historiques avec des questions d'image : l'histoire de l'Europe au $\mathrm{xx}^{\mathrm{e}}$ siècle offre, avec le cas de la France et de l'Allemagne, une parfaite illustration d'une alliance pragmatique qui coexiste avec des images contradictoires.

22. Hummel $(1999,481)$ note que ce syntagme est l'un des rares qui apparaissent plus de trois fois chez Pindare. Avec ses 11 occurrences, il est non seulement le plus fréquent mais il est en outre le seul à contenir un toponyme.

23. Hérodote, 9, 69. Cf. en particulier Sevieri (1999).

24. On trouve une étude détaillée portant sur l'usage des éléments thébains à la gloire de Thèbes dans cette ode chez Sevieri (2000).

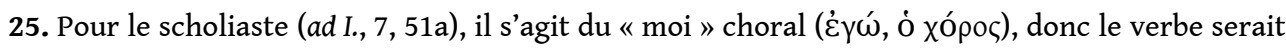
au singulier. On trouve la même position par exemple chez Bury $(1892,131)$, Puech $(1961,70)$, Privitera $\left(2001^{4}, 222\right)$. Wilamowitz $(1922,411)$ considère qu'il s'agit d'un pluriel.

26. Bowra $(1964,153)$, Schachter $(2016,205)$.

27. Les circonstances politiques ont évolué : si l'on admet la datation basse du texte, le témoignage de Thucydide $(1,108)$ montre que Thèbes était alliée de Sparte lors de la bataille de Tanagra. Cf. la « césure » observée après -470 , infra.

28. Outre un poème antérieur aux guerres médiques $(P ., 10)$, on laisse de côté deux poèmes contenant chacun une mention furtive des Héraclides ( $P ., 1,63$ : la mention des Héraclides apparaît dans le cadre d'un éloge des lois doriennes ; P., 5, 71 : Apollon a guidé les descendants d'Héraclès et d'Aigimios, et Pindare chante Sparte).

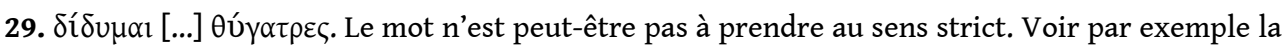
note de Puech $\left(1961^{3}, 77\right.$, n. 1). Il est intéressant de noter que Pindare n'a pas évoqué cette parenté dans les odes qu'il a écrites pour des vainqueurs éginètes avant la fin des guerres médiques (N. 7 -485; N. 5 -483; N. 7 -485; I. 6 -480). Cf. $3^{\text {e }}$ partie de cet article.

30. Il est à noter que cette ode ne contient aucune allusion au glorieux passé récent d'Égine lors de la bataille de Salamine. Évoquer un passé récent ne fait pas l'affaire de Thèbes : Bury (1892, 133-134), Wilamowitz $(1922,197)$, Privitera $\left(2001^{4}, 117\right)$.

31. Carey $(1981,193-195$ et 205) pour le parallèle avec la situation de l'époque.

32. Hérodote, 8, 91-93.

33. On a observé à ce propos que Pindare se garde bien de nommer les Perses, cf. Wilamowitz $(1922,204)$.

34. Zéthos et Amphion, cf. Hésiode, cat. fr. 182-183. La célébrité des aulètes thébains se rattache au personnage de Clonas, cf. Plutarque, De musica, 3 et $5=$ Mor., 1132-1133 et e.g. Roesch (1989). La même remarque pourrait s'appliquer ailleurs (e.g. 0., 3, 8-10; N., 9, 8). Pour la conjonction de la phorminx et de l'aulos, cf. Privitera $\left(2001^{4}, 194\right)$.

35. Pour une discussion sur le rapport éventuel de ces vers avec les luttes entre Agrigente et Syracuse, cf. Gentili et al. (2013, 392), et Schol. ad 0., 2, 29c-d.

36. Farnell $(1932,14-15)$ fait observer que, sans doute par respect pour Théron, Pindare ne parle ni des incestes ni des malédictions que comporte l'histoire des Labdacides. 
37. Sur le fonctionnement de la séquence malheur-bonheur dans cette ode, cf. Hurst (1981, 125-126).

38. Sur les thèmes évoqués dans cette représentation, ainsi que sur la défense de la lecture de võ $\sigma \circ \varsigma$ comme singulier, cf. Gentili et al. $(2013,405)$.

39. Cf. Hurst (1985, 168-169), Lloyd-Jones (1985, 259).

40. Sur le sentiment de solidarité entre le Thébain Pindare et Théron à travers l'évocation de la matière de Thèbes, cf. Wilamowitz (1922, 243-244).

41. Voir par exemple la position prudente de Puech (1931, 49-51).

42. Sur le rapport plus particulier d'Héraclès avec Agrigente, cf. Gentili et al. (2013, 82).

43. Gildersleeve $(1885,156)$ : "The parallel with Herakles is revealed at the end. "

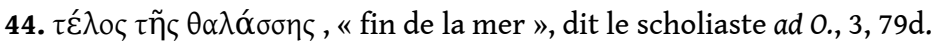

45. Sur ce thème, cf. Farnell (1932, 256-257).

46. Un épisode qui pourrait cependant, par contraste, rappeler la légende de la fondation des jeux de Némée comme jeux funèbres d'Opheltès/Archémoros, bébé tué par un serpent (Apollodore, Bibliothèque, 3, 6, 4). Pour la question de l'adéquation de ce récit à la victoire de Chromios, cf. e.g. Bury (1890, 4-8), Carey (1981, 118-120), Braswell $(1992,56)$.

47. On songe ici à Lefkowitz (1991).

48. Bury $(1890,53)$, pense qu'Héraclès n'est pas nommé pour éviter qu'il n'éclipse la gloire de

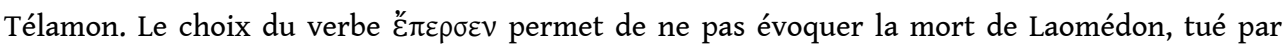
Héraclès.


43-47).

50. Puech $\left(1955^{3}, 39-40\right)$. Carey $(1981,23)$ propose $-477 /-476$. Gentili et al. $(1995,43-47)$ proposent -470 à Delphes ou -468 à Olympie.

51. Cf. Schol. ad P., 2, 6b. Gentili et al. (1995, 366).

52. Il y aurait cependant une forme de «violence " à nommer Thèbes sans nommer le lieu de la victoire remportée par Hiéron selon Wilamowitz $(1922,285)$ (« Gewaltstreich »).

53. E.g. Carey (1981, 50-54, 62-64).

54. E.g. Burton (1962, 111-134) et en particulier la remarque : "This difficult poem is bound to leave an impression of mystery » (133).

55. Schol. ad 0., 10, 101.

56. Schol. ad 0., 10, 19a-b. Scepticisme à ce sujet chez Gentili et al. (2013, 562).

57. On a pu considérer qu'il s'agissait d'une « personal letter of condolence » (Burton, 1962, 78).

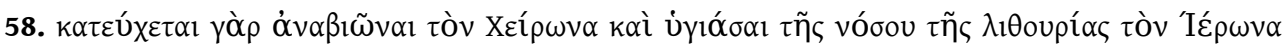
(Schol. ad P., 3, init.).

59. «Una maniera elegante per menzionare Eracle e Tebe e per scusarsi della stravagante citazione » (Gentili et al., 1995, 611). Cette observation implique bien que l'arrivée de Thèbes dans le poème ne va pas de soi.

60. Gentili et al. $(1995,609)$ : «La "digressione" si spiega come omaggio alla propria città. » Discussion chez Burton (1962, 50-56), qui considère que « cette cité » désigne Cyrène. Même position chez Carey $(1981,94)$.

61. On sait que le classement des trois dernières odes «néméennes" est arbitraire : elles célèbrent en fait des victoires obtenues à Sicyone, Argos et Tenedos. On suppose qu'elles constituaient une sorte d'appendice à la fin d'un codex dans lequel les Néméennes figuraient en dernière place, ce qui aurait conduit à les classer finalement parmi les Néméennes malgré leur contenu.

62. E.g. Braswell (1998, 100-101).

63. Dès -470 , date de la première Pythique, Pindare semble parfaitement à l'aise pour évoquer les guerres médiques (P., 1, 75-78).

64. Combats simultanés ou séparés : Farnell (1932, 68-70), Gentili et al. (2013, 530). 
65. Schol. ad 0., 7, init. se référant à Gorgôn, auteur d'un traité sur les sacrifices rhodiens (Athénée, 15, 697a).

66. Sur cette problématique, cf. e.g. Puech $\left(1955^{2}, 61-62\right)$, Schubert (2004, 23-24), Hurst (2005).

67. Henry $(2005,90)$.

68. Pour la justification du choix de ce sujet, alors que s'agissant d'Égine on attendrait les Éacides, cf. Gentili et al. $(1995,573)$.

69. E.g. Burton (1962, 191-193), Burnett (2005, 236-238).

70. La liste comporte, par ordre chronologique : -498 P. 10 / -490 P. 6 / -490 P. 12 / -488 0.14 / -486 P. 7 / -485 N. 2 / -485 ? N. 7 / -483 ? N. 5 / -480 I. 6.

71. Hérodote, 9, 1, 58. Gentili et al. (1995, 263-265).

72. La date de cette épinicie est notoirement difficile à établir. Burton $(1890,114)$ l'établit à -461, mais Farnell (1932, 290), désapprouve : «The long introduction in Bury's edition is a salient example of the false method of interpreting Pindar. " Wilamowitz $(1922,160)$ propose la période entre -490 et -485. Radt (1958, 90-93) se rallie à l'idée d'une « période moyenne » de Pindare, soit -478/-460.

73. La découverte du sixième Péan de Pindare a relancé l'intérêt pour la septième Néméenne : e.g. Wilamowitz (1908), Puech (1923, 85-87), Radt (1958, 85-88), Carey (1981, 133-137), Rutherford (2001, 185-202), Burnett (2005, 185-202).

74. Pour le contenu de ces exploits, cf. Farnell (1932, 360), Privitera (20014, 207-208).

75. Une scène dont l'effet pourrait se voir augmenté par le fait qu'elle reproduit un schéma connu : la visite à l'improviste d'un dieu qu'il s'agit d'accueillir favorablement pour éviter des conséquences désastreuses. Burnett (2005, 84-85).

76. "Kein Wort deutet auf die Gefahr, die von Asien droht, und doch ist das Heer des Xerxes längst unterwegs ", écrit à ce sujet Wilamowitz $(1922,181)$. On aurait peut-être cette allusion dans la scène de la rencontre d'Héraclès et de Télamon et son invitation à combattre en Asie.

\section{RÉSUMÉS}

Après la fin des guerres médiques, alors que Thèbes a pris le parti des envahisseurs, Pindare va recourir dans ses épinicies à diverses stratégies pour défendre l'image ternie de sa cité. Si la glorification de Thèbes constitue une partie obligée des éloges de vainqueurs thébains, il n'en va pas de même pour les épinicies qui célèbrent des vainqueurs venus d'autres cités grecques. Les manières diversifiées d'y introduire frontalement ou obliquement des thématiques thébaines semblent bien traduire un souci du poète pour la renommée de Thèbes. On observe en outre une nette différence dans le traitement de thèmes thébains entre les épinicies composées avant et après la fin des guerres médiques.

After the end of the Persian wars, and Thebes having sided with the invaders, Pindar, in his victory odes, uses several strategies aimed at vindicating the tarnished image of his city. To glorify Thebes might be a regular part of every ode celebrating a victory won by a Theban, but this cannot be said of odes glorifying champions of other cities. The different procedures, direct or oblique, used by Pindar in order to introduce Theban themes seem to indicate a preoccupation of the poet with the reputation of Thebes. Moreover, one can observe a difference between odes composed before and after the end of the Persian wars in the use made of themes related to Thebes. 
INDEX

Mots-clés : Pindare, épinicies, image de Thèbes, guerres médiques

Keywords : Pindar, victory odes, Thebes' image, Persian wars

\section{AUTEUR}

\section{ANDRÉ HURST}

Université de Genève.

Professeur honoraire et ancien recteur de l'université de Genève, André Hurst a publié des travaux notamment dans les domaines de la poésie hellénistique, du théâtre antique, de l'historiographie grecque et de la papyrologie. Il poursuit actuellement des recherches sur Pindare (il avait en 1984 préparé et présidé les « Entretiens sur l'Antiquité classique de la Fondation Hardt » sur Pindare, vol. XXXI, 1985). 